\title{
DESCRIPCIÓN DE UNA NUEVA ESPECIE DE THORECTES MULSANT, 1842 DEL NORTE DE MARRUECOS (COLEOPTERA, SCARABAEOIDEA, GEOTRUPIDAE)
}

\author{
J. L. Ruiz (*)

\section{RESUMEN}

Se describe una nueva especie de Thorectes Mulsant del subgénero Thorectes, procedente del Rif noroccidental (noroeste de Marruecos): T. (T.) coloni n. sp. Se establecen, con fines prácticos y en base a caracteres morfológicos externos, dos grupos de especies norteafricanas en el seno del subgénero Thorectes: uno constituido por Thorectes laevigatus y T. trituberculatus y otro por T. armifrons y T. coloni $\mathrm{n}$. sp. Se discuten las diferencias y afinidades entre T. coloni $\mathrm{n}$. sp. y las demás especies que componen el subgénero Thorectes.

Palabras clave: Coleoptera, Scarabaeoidea, Geotrupidae, Thorectes, nueva especie, Marruecos.

\begin{abstract}
Description of a new species of Thorectes Mulsant, 1842 from North Morocco (Coleoptera, Scarabaeoidea, Geotrupidae)

Thorectes coloni, a new species in the subgenus Thorectes Mulsant from Rif mountains (northwestern Morocco) is decribed. Two groups of North African species in the subgenus Thorectes are stablished for practical purposes based on external morphological traits: one group including T. laevigatus and T. trituberculatus and the other group containing T. armifrons and T. coloni. Differences and similarities between T. coloni and the other species in the subgenus Thorectes are discussed.
\end{abstract}

Key words: Coleoptera, Scarabaeoidea, Geotrupidae, Thorectes, new species, Morocco.

\section{Introducción}

El género Thorectes Mulsant, 1842 ha sido recientemente escindido por López-Colón (1996) en cinco géneros, Thorectes Mulsant, 1842, Baraudia López-Colón, 1996, Silphotrupes Jekel, 1866, Zuninoeus López-Colón, 1989 y Jekelius, López-Colón, 1989, que vendrían a corresponderse con otras tantas líneas filogenéticas en el seno del antiguo género, poniendo de manifiesto el polifiletismo del grupo, parecer ya esbozado por Krikken
(1981) y Zunino (1984). En contraposición a lo expresado por Baraud (1965, 1966, 1985, 1992), el estudio detallado de la genitalia masculina se ha revelado como esencial en la sistemática interna del grupo, ya sea a nivel genérico o infragenérico (López-Colón, 1989, 1995, 1996; López-Colón y Romero Samper, 1996).

Dentro del género Thorectes sensu López-Colón (1996) este autor diferencia, atendiendo a caracteres tanto genitales como de morfología externa, dos subgéneros: Thorectes Mulsant, 1842 y Renaudia

\footnotetext{
* Avda. Madrid, 4, $1^{\circ}$ izq., 51001 Ceuta (España).
} 
López-Colón, 1996. En el presente trabajo se describe una nueva especie de Thorectes del subgénero Thorectes, a partir de tres ejemplares colectados en el transcurso de diversos muestreos faunísticos encaminados a documentar la diversidad coleopterológica de la pequeña Sierra del Haus (noroeste de Marruecos).

\section{Material y métodos}

El material típico fue obtenido mediante muestreos directos de fauna epígea, colectándose uno de los individuos bajo una piedra, otro directamente en el suelo y un tercero ahogado en una charca temporal. El estudio morfológico de los ejemplares fue realizado de la manera habitual en Scarabaeoidea de mediano o gran tamaño, mediante observación con estereomicroscopio, en este caso un Olympus SZH-10. Las genitalias y segmentos genitales una vez extraídos se montaron en cartulinas con resina D.M.H.F., las cuales se pincharon con su respectivo ejemplar. Las medidas efectuadas se llevaron a cabo con un micrómetro Olympus 26 OCM10/100XY acoplado a uno de los oculares. Los dibujos se efectuaron con cámara clara. La nomenclatura de las diferentes partes de la genitalia masculina se basa en la expresada por López-Colón (1989) y López-Colón y Romero Samper (1996).

\section{Thorectes (Thorectes) coloni n. sp.}

HoLотүрus: 1 macho, etiquetado: "Tleta-Taghramt, Anyera, Rif, Marruecos, 25-I-98, J.L. Ruiz leg.” (etiqueta blanca, manuscrita e impresa); "Holotypus, Thorectes (Thorectes) coloni, J.L. Ruiz des. 1998" (etiqueta roja, manuscrita e impresa). Depositado en la colección del Museo Nacional de Ciencias Naturales de Madrid (España).

PARATYPI: 1 macho, etiquetado: "Yebel Musa, Anyera, Rif, Marruecos, 14-XII-97, F.J. Martínez leg." (etiqueta blanca, manuscrita e impresa), en col. J.L. Ruiz (Ceuta, España); 1 hembra, etiquetada: "Tleta-Taghramt, Anyera, Rif, Marruecos, 23-XI-97, J.L. Ruiz leg." (etiqueta blanca, manuscrita e impresa), en col. Museo Nacional de Ciencias Naturales de Madrid (España). Ambos ejemplares llevan la siguiente etiqueta: "Paratypus, Thorectes (Thorectes) coloni, J.L. Ruiz des. 1998" (etiquetas rojas, manuscritas e impresas).

DESCRIPCIÓN DEL HOLOTIPO (MACHO): Longitud: 19,8 mm. Anchura máxima, situada a nivel del primer tercio elitral: $11,9 \mathrm{~mm}$. Coloración totalmente negra, sin ningún tipo de reflejo azulado o de cualquier otra tonalidad. Aspecto muy brillante. Forma general convexa.

Cabeza (fig. 1) brillante, con el clípeo semicircular, ligeramente realzado en el borde externo, con un punteado fuerte, confluente y subvermiculado, ofreciendo un aspecto rugoso. Tubérculo cefálico muy fuerte y alzado, cónico con el vértice redondeado, situado un poco por detrás de la mitad clipeal (relación $\mathrm{a} / \mathrm{b}=1,35$; ver fig. 1 ), elevado sobre el plano clipeal $0,8 \mathrm{~mm}$ y prolongado hacia delante en una débil carena de límites difusos. Sutura clípeo-frontal arqueada, sutilmente marcada. Frente y vértex con punteado menos fuerte que el del epistoma, de aspecto subvermiculado. Bordes laterales de la frente, a ambos lados de la región anterior de los ojos, realzados, casi tuberculiformes. Región posterior del vértex prácticamente impunteada. Mejillas semicirculares, salientes, bien separadas del borde del clípeo. Mandíbulas no bilobuladas en su arista externa, sólo con una ligera sinuación por detrás del lóbulo distal que sí es marcado y saliente en la mandíbula derecha y mucho menos evidente en la izquierda.

Pronoto 1,62 veces más ancho que largo, con la máxima anchura a nivel del tercer cuarto, presentando una neta foseta anterocentral hemielíptica que alcanza el primer tercio pronotal. Angulos anteriores obtusos, los posteriores ampliamente redondeados. Totalmente rebordeado, con dicho reborde muy grueso en el margen anterior y débil en dos 


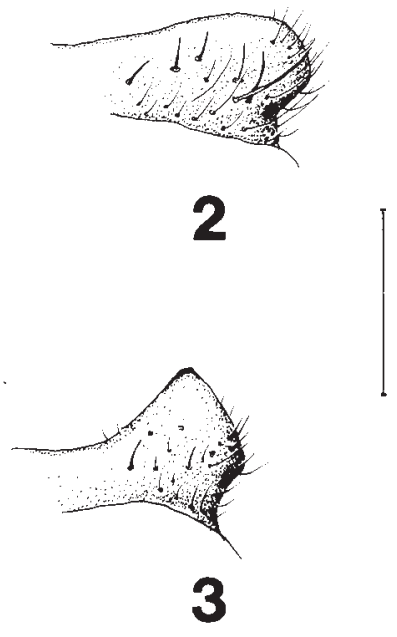

Figs. 2-3.- Apófisis mesosternal en visión lateral: 2) Thorectes coloni $\mathrm{n}$. sp. 3) Thorectes armifrons. (Escala: 0,5 mm).

Figs. 2-3.- Mesosternal apophysis in lateral view: 2) Thorectes coloni n. sp. 3) Thorectes armifrons. (Scale: 0,5 mm).

pequeñas zonas laterales de la base, situadas entre la cuarta y séptima estrías elitrales, pero sin llegar a borrarse. Punteado pronotal muy escaso, nada confluente, doble, constituido por unos puntos fuertes, netos y umbilicados y otros más finos, la mitad de diámetro que los anteriores y mezclados difusamente entre aquellos, distribuidos por la foseta anterior, laterales y ángulos anteriores, así como en una línea junto al reborde basal, separados en estas zonas entre 1,5 y 5 veces su diámetro. Disco pronotal prácticamente impunteado, sólo con escasos puntos finos dispersos.

Escudete brillante, triangular, transverso, doble de ancho que largo, impunteado.

Élitros convexos, brillantes pero algo menos que el pronoto debido a una finísima y casi imperceptible microrreticulación; con escasas y ligeras arrugas transversas. Punteado limitado exclusivamente a las estrías elitrales, que aparecen definidas por líneas más o menos subparalelas de puntos netos de tamaño similar o algo mayor que los pequeños pronotales. Reborde marginal de los élitros realzado en todo su recorrido, interrumpiéndose una vez pasada la región humeral a partir de donde la base de los élitros aparece muy débilmente realzada en una fina línea.

Apófisis mesosternal (fig. 2) apenas elevada y prolongada hacia delante, con su extremo anterior enteramente redondeado.
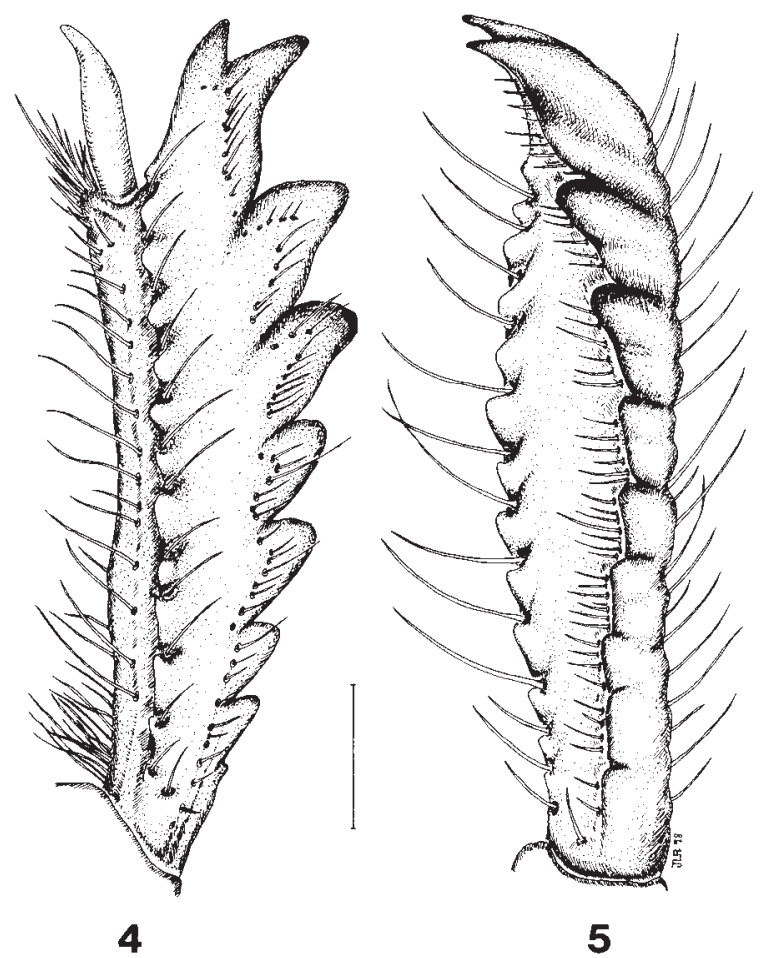

Figs. 4-5.- Thorectes coloni n. sp., protibia izquierda del macho: 4) Visión ventral. 5) Visión lateral. (Escala: $1 \mathrm{~mm}$ ).

Figs. 4-5.- Thorectes coloni n. sp., left anterior tibia of the male: 4) Ventral view. 5) Lateral view. (Scale: $1 \mathrm{~mm}$ ).

Esternitos abdominales brillantes, con un microrreticulado finísimo similar al de los élitros, sin rugosidad alguna; presentando cada esternito una línea transversa irregular de puntos pilíferos en el medio de la mitad distal y algunos puntos hacia los lados, en la mitad proximal.

Tibias anteriores con ocho dientes en su margen lateral externo (figs. 4 y 5); siendo el basal muy pequeño y el apical marcadamente bifurcado. Tercer diente (contado desde el ápice) situado en el mismo plano que los demás. Arista inferointerna con una serie continua de doce dientes poco sobresalientes, separados cada uno de ellos por una foseta pilífera. Superficie inferior de las protibias brillante, apenas subrugosa y con una microrreticulación casi imperceptible.

Fémures posteriores anchos, muy brillantes, con la superficie casi lisa, sólo con algunos muy pequeños puntos dispersos (fig. 6). Carena anteroventral débil y sinuosa, constituida por una línea de puntos pilíferos granulosos. Carena ventral posterior ine- 


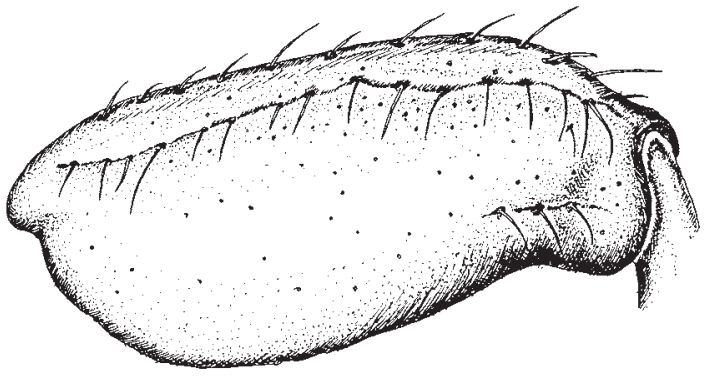

6

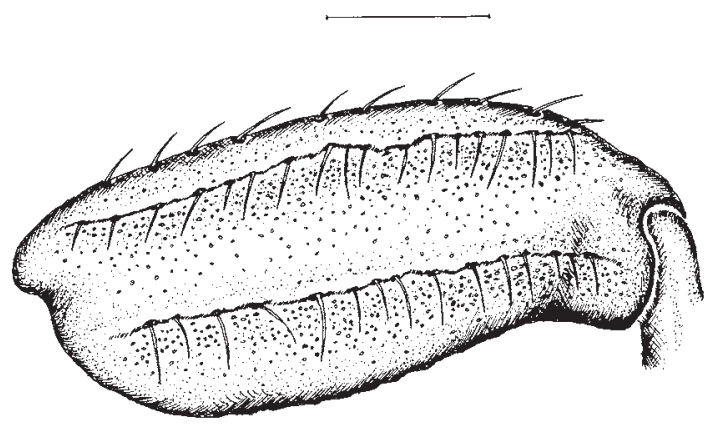

7

Figs. 6-7.- Cara ventral del fémur posterior izquierdo del macho: 6) Thorectes coloni n. sp. 7) Thorectes armifrons. (Escala: $1 \mathrm{~mm}$ ).

Figs. 6-7.- Ventral side of left posterior femur of the male: 6) Thorectes coloni $\mathrm{n}$. sp. 7) Thorectes armifrons. (Scale: $1 \mathrm{~mm}$ ).

xistente, sólo esbozada por dos o tres puntos setígeros en el cuarto distal. Arista posterior prácticamente lisa, con muy ligeras denticulaciones.

Edeago: figs. 8 y 9. Región apical del parámero izquierdo ensanchada, con un diente no muy fuerte pero bien patente, montado sobre la zona subapical parameral derecha, que a su vez se ensancha hacia el extremo distal. Desesclerificación del margen medio dorsal del parámero izquierdo de escasa superficie, hemielíptica, sin ángulos marcados en el lado interno. Arco basal dorsal de los parámeros de contorno redondeado. Lóbulo izquierdo del arco basal dorsal fuerte y subtrapezoidal. Desesclerificación del arco basal dorsal profunda y escotada. Escotadura central de la cara dorsal de la falobase alcanzando un poco más de la mitad, con el vértice basal marcado en ángulo agudo. Falobase ancha y poco incurvada en visión lateral. Lóbulos dorsales de la falobase bastante prolongados hacia el ápice. Surcos interlobulares bien manifiestos. Lóbulo medio con la inserción de las láminas medianas separadas y regularmente entrecruzadas en aspa en el medio.

HEMBRA: La única hembra estudiada, que se encuentra bastante deteriorada, mide $21,7 \mathrm{~mm}$ y es muy similar al macho, del que se diferencia por los siguientes caracteres: aspecto un poco más ancho y robusto; pronoto 1,74 veces más ancho que largo; foseta antemedia del pronoto menos hundida; punteado pronotal un poco más denso, tanto en la foseta como hacia los lados y disco; líneas de puntos de las estrías elitrales más marcadas; diente apical externo de las tibias anteriores simple, no bifurcado; carena inferointerna de las protibias con una fila de dieciseis dientes cortos, de entre los que el sexto, octavo y onceavo (contados desde la base) sobresalen ligeramente del resto $\mathrm{y}$, por último, fémures posteriores con una línea de seis puntos pilíferos en la región ventral posterior que ocupan el tercio distal.

VARIABILIDAD: La variabilidad se refiere, en este caso, a un único paratipo macho. Este presenta una longitud algo menor que el holotipo, $18,9 \mathrm{~mm}$; aspecto general aún más brillante debido al casi nulo desgaste tegumentario; pronoto 1,63 veces más ancho que largo; punteado pronotal algo más escaso y protibias anteriores con catorce dientes subiguales en su arista inferointerna, siendo en el resto de caracteres prácticamente similar al Holotipo.

DISTRIBUCIÓN Y HÁBITAT: Thorectes coloni únicamente se conoce de dos localidades muy cercanas, distanciadas entre sí tan solo $15 \mathrm{~km}$, en la extensa comarca de Anyera (fig. 12): Tleta-Taghramt (altitud: 420 m; cuadrícula U.T.M. 10x10: 30STE86) y Yebel Musa (altitud máxima: $842 \mathrm{~m}$; cuadrícula U.T.M. 10x10: 30STE87), situadas en el extremo noroccidental de la Península Tingitana (Gharbaoui, 1981). Tleta-Taghramt se ubica en la Sierra del Haus (eje longitudinal: $35 \mathrm{~km}$ aproximadamente), relieve montañoso conformado por una alineación de crestas en sentido norte-sur con alturas que rondan los $500 \mathrm{~m}$ (mayor altitud: Yebel Haus, $840 \mathrm{~m}$ ). El bloque del Yebel Musa, en contacto con el mar por su cara norte, forma parte de la llamada Sierra Bullones, que no es sino una pequeña continuación geográfica hacia el noroeste de la Sierra del Haus. Desde el punto de vista geológico, 


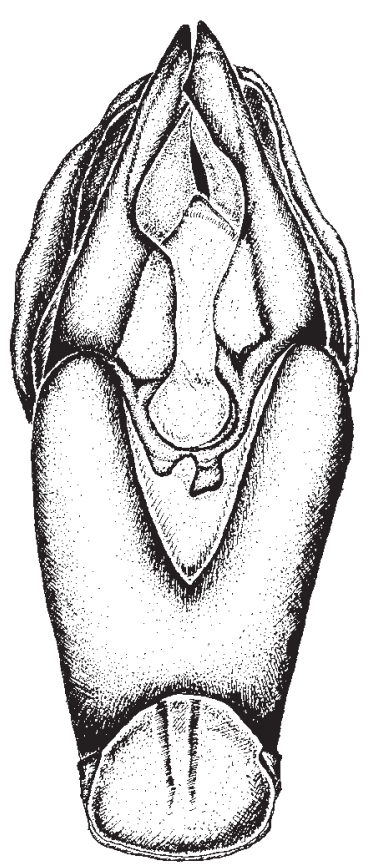

8

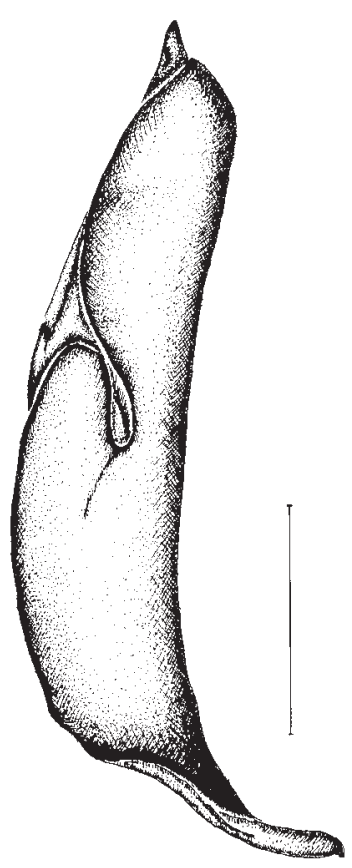

9

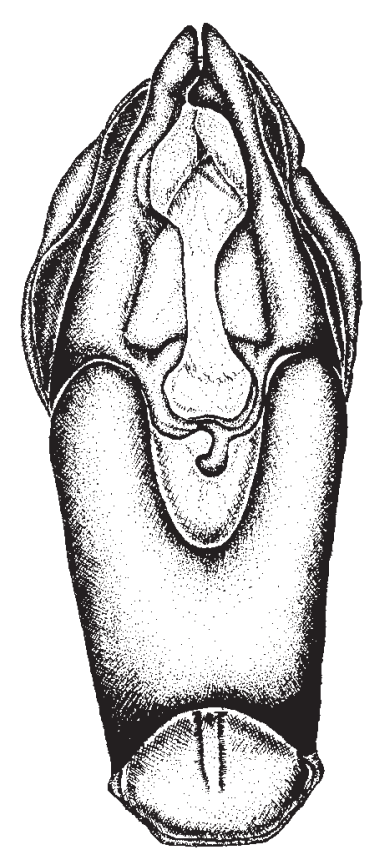

10

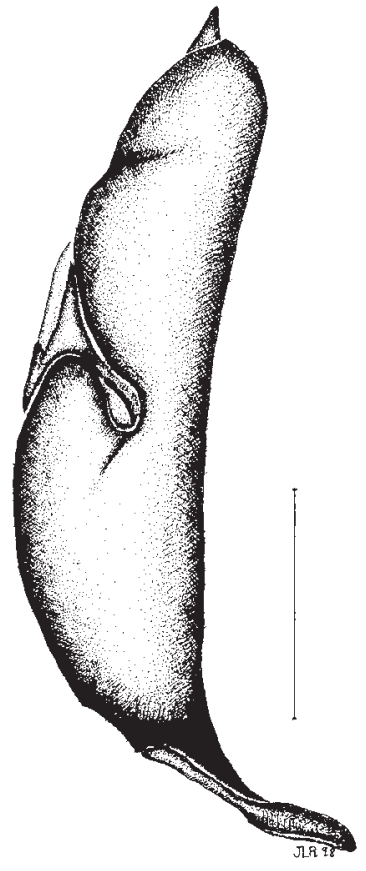

11

Figs. 8-11.- 8-9) Thorectes coloni n. sp.: 8) Edeago en visión dorsal. 9) Edeago en visión lateral. 10-11) Thorectes armifrons: 10) Edeago en visión dorsal. 11) Edeago en visión lateral. (Escala: $1 \mathrm{~mm}$ ).

Figs. 8-11.- 8-9) Thorectes coloni n. sp.: 8) Aedeagus in dorsal view. 9) Aedeagus in lateral view. 10-11) Thorectes armifrons: 10) Aedeagus in dorsal view. 11) Aedeagus in lateral view. (Scale: $1 \mathrm{~mm}$ ).

tanto la Sierra del Haus como el Yebel Musa se ubican en el dominio estructural de la Dorsal caliza Rifeña, unidad constituida por una potente formación caliza mesozoica que origina un relieve agreste con un modelado típicamente kárstico (Michard, 1976; Chamorro y Nieto, 1989), siendo los materiales que las conforman dolomías triásicas y calizas liásicas en el Haus y dolomías del Liásico inferior en el caso del Yebel Musa (El Hatimi et al., 1991; Chamorro, 1995). Sendas localidades se encuadran en el piso termomediterráneo, con un bioclima de tipo húmedo templado (Benabid, 1982, 1984). La vegetación asentada en estas dos zonas es prácticamente similar $\mathrm{y}$, en general, muestra un aspecto achaparrado y ralo, consecuencia de la naturaleza rocosa del terreno, los fuertes y constantes vientos que azotan la región y el sobrepastoreo. La asociación vegetal presente mayoritariamente es la denominada Saturejo graecae-Coridothymetum capitati Quézel, Barbéro, Benabid, Loisel y RivasMartínez, 1988 (Quézel et al., 1988; Benabid y
Fennane, 1994). Como elementos florísticos característicos podemos destacar: Pistacia lentiscus L., Ulex eriocladus C. Vicioso, Crataegus monogyna Jacq., Chamaerops humilis L., Rhamnus oleoides (L.) Jahand et Maire, Mentha pulegium L., Teucrium polium L., Teucrium scorodonia L., Bupleurum balansae Pau, Stachys fontqueri Pau y Viola reichenbachiana Jord.

Como dato ecológico, señalar que en TletaTaghramt y en las mismas fechas de captura de los especímenes tipo, se encontraron numerosos restos (pronotos, patas, élitros) de T. coloni en egagrópilas de chova piquirroja [Pyrrhocorax pyrrhocorax barbarus (Gmelin, 1774)], córvido abundante en la zona.

Etimología: Especie que tenemos el placer de dedicar a nuestro querido amigo y gran entomólogo D. José Ignacio López-Colón, artífice de la actual taxonomía de Thorectes entre otras muchas y meritorias investigaciones, en términos de recono- 


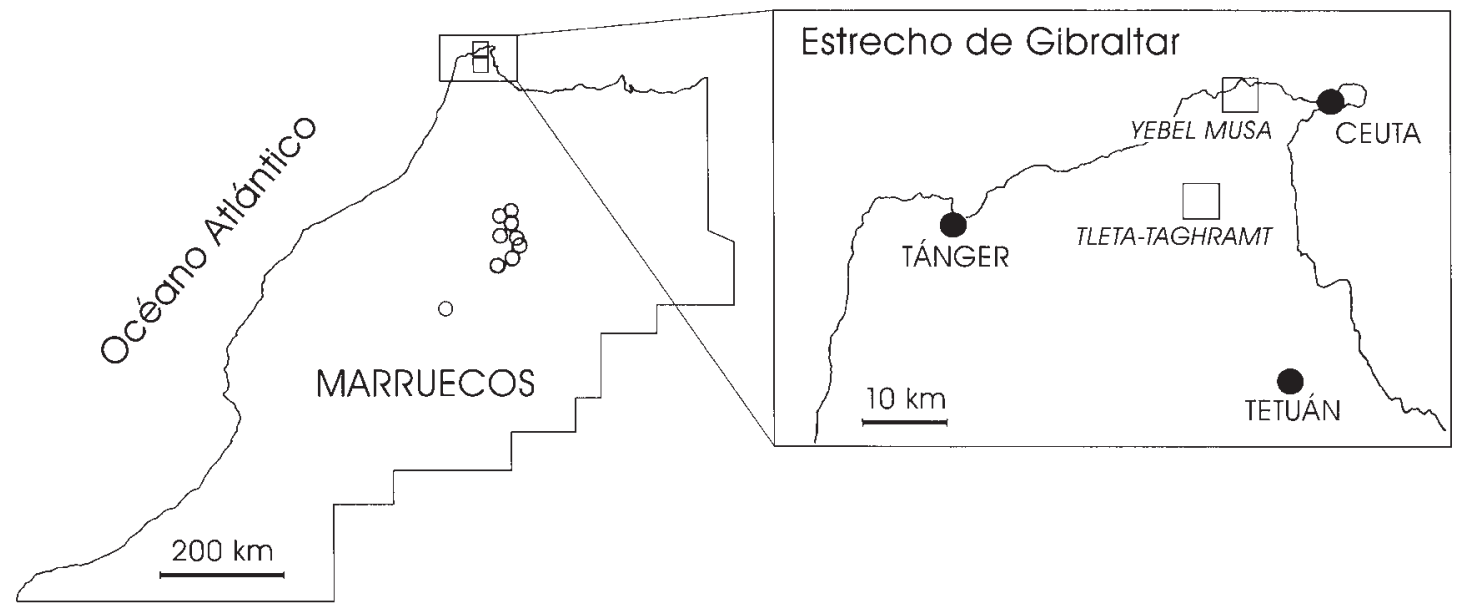

Fig. 12. - Mapa de Marruecos mostrando las localidades conocidas de Thorectes coloni n. sp. (cuadrados blancos) y Thorectes armifrons (círculos blancos).

Fig. 12.- Map of Morocco showing the known localities of Thorectes coloni n. sp. (white squares) and Thorectes armifrons (white circles).

cimiento y gratitud por su continua ayuda y enseñanzas.

\section{Discusión}

En el marco de la nueva ordenación taxonómica del género Thorectes Mulsant, 1842 sensu lato propuesta por López-Colón $(1989,1996)$ y LópezColón y Romero Samper (1996), en la que cobra especial relevancia la morfología de la armadura genital masculina, Thorectes coloni $\mathrm{n}$. sp. se integra de manera evidente en el género Thorectes sensu López-Colón (1996), caracterizado por tener el edeago manifiestamente ensanchado en el medio, la falobase fuertemente escotada en el centro de la cara dorsal, el arco basal dorsal de los parámeros más o menos lobulado o engrosado, los parámeros con los márgenes mediodorsales bien desarrollados y el lóbulo medio con la inserción de las láminas medianas separadas y regularmente entrecruzadas en aspa (López-Colón, 1996).

Dicho género ha sido dividido a su vez en dos subgéneros (López-Colón, 1996), subgén. Thorectes s. str. (especie-tipo: Scarabaeus laevigatus Fabricius, 1798) y subgén. Renaudia LópezColón, 1996 (especie-tipo: Thorectes distinctus Marseul, 1878). Thorectes coloni $\mathrm{n}$. sp. comparte los caracteres discriminatorios del primero de estos subgéneros, en el cual queda agrupado, y que son los siguientes: escotadura central de la cara dorsal de la falobase llegando aproximadamente hasta la mitad; desesclerificaciones de los márgenes mediodorsales de los parámeros bien desarrolladas por detrás, ápice de los parámeros denticulado en la cara interna; especies bastante o muy convexas, con el pronoto menor o de igual anchura que los élitros; ángulos posteriores del pronoto ampliamente redondeados, no aplicados contra la base elitral; base de los élitros con un reborde lateral que no se prolonga hacia el escudete.

El subgénero Thorectes se distribuye por la mitad sur de la Península Ibérica (España y Portugal) y norte de África (Marruecos y Argelia) (Baraud, 1965, 1966, 1977, 1985, 1992; Báguena, 1967; López-Colón, 1981, 1983, 1989, 1990, 1996; López-Colón y Romero Samper, 1996; LópezColón et al., 1997; Ruiz et al., 1995) e integra, por el momento, a las siguientes especies: Thorectes lusitanicus (Jekel, 1866) [= Thorectes laevigatus cobosi (Baraud, 1966)], Thorectes valencianus (Baraud, 1966), Thorectes baraudi López-Colón, 1981, Thorectes ferreri López-Colón, 1983, Thorectes laevigatus (Fabricius, 1798), Thorectes trituberculatus (Reitter, 1893), Thorectes armifrons (Reitter, 1893) y Thorectes coloni n. sp. Las cuatro primeras son exclusivas de la mitad meridional de la Península Ibérica y las cuatro restantes sólo se 
encuentran, con un areal de distribución más o menos restringido, en el Magreb occidental.

Ciñéndonos a la representación específica norteafricana del subgénero Thorectes podemos diferenciar, con fines meramente prácticos, dos grupos de especies que no necesariamente deben evidenciar líneas filéticas reales. El primer grupo estaría constituido por T. laevigatus y $T$. trituberculatus y vendría caracterizado por presentar ambas especies un tubérculo cefálico más bien débil y de inserción posterior, cercana a la sutura clípeo-frontal; las mandíbulas marcadamente bilobuladas en el borde externo (carácter fácilmente observable en ejemplares poco "rozados"); las tibias anteriores de los machos con el tercer diente lateral externo (contado desde el ápice), más inclinado hacia abajo que los demás; y la carena inferointerna de las protibias de los machos con una serie de dientes alineados a modo de sierra de entre los cuales dos o tres, situados entre el tercero y quinto externos, son netamente mayores sobresaliendo sobre el resto (Reitter, 1893; Baraud, 1965, 1966, 1985; Ruiz et al., 1995).

El segundo grupo lo integrarían T. armifrons y T. coloni y se diferencia del anterior por la conjunción de los caracteres que siguen: tubérculo cefálico elevado, casi centrado en la mitad del clípeo; mandíbulas nada o apenas bilobuladas en su borde externo; tibias anteriores del macho con el tercer diente lateral externo en el mismo plano que el resto y carena inferointerna de las protibias del macho con una serie de dientes pequeños alineados de aproximadamente igual tamaño, sin que ninguno sea marcadamente mayor que el resto (Reitter, 1893; Baraud, 1965, 1985). Esto último se contrapone en parte a lo expresado por Baraud (1965, 1985), quien indica que T. armifrons muestra la conformación de la arista inferointerna de las protibias del macho análoga o del mismo tipo que T. trituberculatus y este a su vez igual que T. laevigatus. Por nuestra parte, hemos estudiado abundante material de T. armifrons (27 machos y 19 hembras; J.M. Ávila leg., P. Barranco leg. y F. Delgado leg.) procedente de Ifrane y Azrou (Medio Atlas, Marruecos), localidades ya señaladas por otros autores para la especie (Kocher, 1958, 1969; Baraud, 1965, 1971, 1985; Dewhurst, 1980), comprobando que realmente este taxón presenta la arista inferointerna de las protibias masculinas constituida por una serie continua de dientes pequeños y subiguales (entre 12 y 16 ; media $=14 ; \mathrm{n}=27$ ). Por contra, el examen de 10 machos (J.M. Ávila leg. y J.M. Guzmán leg.) identificados como T. trituber- culatus procedentes de la región de Taza (noreste de Marruecos) nos muestra que, en efecto, esta especie sí presenta la conformación de las tibias anteriores del macho del mismo tipo (aunque con algunas diferencias) que T. laevigatus (caracterizado en Ruiz et al., 1995), tal como hemos expresado en la segregación morfológica de estos dos grupos.

De esta forma, $T$. coloni sería una especie morfológicamente afín a $T$. armifrons. Las diferencias morfológicas externas entre ambas son las siguientes: aspecto más convexo y brillante en T. coloni, debido esto último a una microrreticulación menos patente en el pronoto y sobre todo en los élitros; tubérculo cefálico más fuerte y elevado en $T$. colo$n i$, con la base más ancha y difusamente prolongado en carena hacia delante; relación "anchura máxi$\mathrm{ma} /$ longitud en el medio" del pronoto comprendida en el macho de $T$. coloni entre 1,62 y 1,63 (media= 1,$625 ; \mathrm{n}=2$ ) y en la única hembra estudiada 1,74 , oscilando esta relación en T. armifrons entre 1,72 y $1,94$ en los machos (media $=1,82 ; n=27)$ y entre 1,77 y 1,94 en las hembras (media $=1,87 ; n=19$ ), lo que se traduce en un aspecto un poco más estilizado del pronoto en visión dorsal de la nueva especie (la validez discriminante de este carácter merístico es, no obstante, accesoria, hasta el estudio de un mayor número de ejemplares de T. coloni); punteado pronotal mucho menos denso en $T$. coloni, circunscrito casi de manera exclusiva a los lados, borde anterior y foseta antemedia, constituido principalmente por fuertes y profundos puntos umbilicados que alcanzan su máxima densidad en los márgenes laterales y foseta antemedia, pero sin llegar en ningún momento a confluir, constituido en $T$. armifrons por puntos menos impresos y apenas umbilicados, subconfluente en los lados y foseta antemedia, invadiendo de forma manifiesta el disco, en conjunto mucho más denso; apófisis mesosternal apenas levantada y prolongada hacia delante en la primera de las especies (fig. 2), fuertemente elevada, más o menos perpendicular al eje del cuerpo y en forma de hacha en la segunda (fig. 3); metafémures con la carena ventral posterior reducida en los machos de $T$. coloni a dos o tres puntos pilíferos en el extremo distal (fig. 6) y en las hembras a seis puntos que ocupan el tercio apical, mientras que dicha carena es entera y neta en ambos sexos de T. armifrons (fig. 7), asimismo esta especie presenta la superficie ventral de los metafémures con un micropunteado relativamente denso y manifiesto, mientras que en la primera es prácticamente lisa y brillante; superficie inferior de las protibias del macho de T. coloni con un micropuntea- 
do muy débil y disperso, presentando T. armifrons dicho micropunteado fuerte y denso; por ultimo, los esternitos abdominales de T. armifrons son fuerte y profundamente micropunteados, y casi lisos, con tal micropunteado débil y disperso en $T$. coloni. El carácter referente a la apófisis mesosternal es empleado por López-Colón (1996) en su clave de géneros, indicando que Thorectes presenta la "apófisis mesosternal más o menos levantada, dirigida hacia abajo, perpendicular al eje del cuerpo", cosa que no ocurre en la nueva especie constituyendo una excepción en el seno del género.

Respecto a la genitalia masculina, las diferencias constatadas entre estas dos especies son:

Thorectes coloni (figs. 8 y 9): mitad apical del parámero izquierdo relativamente ancha, aplanada dorsalmente, con un débil y poco patente diente en su región apical. Parámero derecho igualmente aplanado en visión dorsal, nada sinuoso, con el borde interno en curva regular y el ápice puntiagudo. Arco basal dorsal de los parámeros redondeado en su borde interno, con el lóbulo derecho sobresaliente. Lóbulos dorsales de la falobase alargados, sobrepasando la inserción basal de las desesclerificaciones medio dorsales de los parámeros. Escotadura apical de la falobase triangular. Falobase poco curvada en visión lateral. Lóbulos ventrales de la falobase sin estrangulamientos laterales.

Thorectes armifrons (figs. 10 y 11): mitad apical del parámero izquierdo estrecha, no aplanada dorsalmente y con un muy fuerte diente en la región apical. Parámero derecho sinuoso, tanto dorsalmente como en su borde interno, con el ápice romo. Arco basal dorsal de los parámeros sinuado en el medio de su borde interno, con el lóbulo derecho débil. Lóbulos dorsales de la falobase sin alcanzar el nivel de las desesclerificaciones mediodorsales de los parámeros. Escotadura apical de la falobase hemielíptica. Falobase bastante curvada en visión lateral. Lóbulos ventrales de la falobase con un estrangulamiento lateral un poco hacia atrás de la mitad.

Las especies ibéricas del subgénero Thorectes se separan sin dificultad de T. coloni por compartir los caracteres anteriormente señalados para el grupo constituido por $T$. laevigatus y $T$. trituberculatus (Baraud, 1965, 1966, 1977, 1985, 1992; LópezColón, 1996; López-Colón y Romero Samper, 1996; Ruiz et al., 1995), con la excepción de $T$. ferreri [considerada por Baraud (1992) sinónima de T. laevigatus cobosi (Baraud, 1966) a tenor de los datos sobre dimorfismo sexual aportados por
Branco (1984) para esta última] que muestra las mandíbulas apenas bisinuadas y la arista inferointerna de las protibias del macho con los dientes subiguales, así como el tercer diente externo protibial en el mismo plano que los demás. Sin embargo, ésta es bien diferente de la nueva especie por su punteado pronotal denso y fuerte incluso en el disco, el diente apical externo de las protibias del macho simple y su particular genitalia masculina, además de presentar el tubérculo cefálico débil y de inserción posterior, la apófisis mesosternal elevada y la carena ventral posterior de los metafémures entera (López-Colón, 1983, 1996; López-Colón y Romero Samper, 1996).

Aún a pesar de los escasos datos corológicos disponibles, T. armifrons y T. coloni parecen mostrar áreas de distribución claramente alopátricas, lo que reforzaría el aislamiento específico entre ambas. Reitter (1893) indicó como locus typicus de $T$. armifrons, Casablanca (costa atlántica marroquí), único registro que es señalado por Escalera (1914). Kocher (1958) y Baraud (1965), aunque recogen dicha localidad, la sitúan repartida por diversos parajes del Medio Atlas. Posteriormente, estos mismos autores (Kocher, 1969; Baraud, 1985) consideran a todas luces errónea la cita de Casablanca pues, como argumenta Kocher (1969), los autores de finales del siglo pasado que estudiaron material marroquí solían designar como localidades típicas aquellas desde donde les eran enviados los ejemplares (Casablanca, Tánger, Mogador) y que, en numerosas ocasiones, no se correspondían con los lugares reales de captura. Así, T. armifrons sería una especie montana propia del Medio Atlas (Azrou, AinKahla, Tannezoult, Ag. Sidi-Ali, Dj. Hebri, Ifrane, Timhadit, Arbalou) a altitudes comprendidas entre 1400 y $2100 \mathrm{~m}$, habiendo sido señalada igualmente del Gran Atlas central (Foret Haouinet, $2200 \mathrm{~m}$ ) (Kocher, 1958, 1969; Baraud, 1965, 1971, 1985) (fig. 12).

Thorectes coloni se conoce únicamente de dos localidades muy cercanas entre sí, en el extremo noroccidental del Rif (fig. 12). Este taxón posiblemente sea endémico de los abruptos relieves calizos de la Sierra del Haus y Yebel Musa (o, por extensión, de la Dorsal caliza Rifeña), suposición que apoyamos en el hecho de no haber sido hallado en localidades próximas intensamente muestreadas, como Ceuta (véase Ruiz, 1995) a tan solo $2 \mathrm{~km}$ del Musa o Tánger, objeto de intensas cazas durante el pasado siglo y comienzos del presente (Escalera, 1914; Kocher, 1956). En este sentido, la pequeña 
sierra del Haus y el Y. Musa constituyen "islas" calizas en el norte de la Península Tingitana (mayoritariamente silicícola) y que, aunque con parámetros climáticos muy semejantes, presentan una fisionomía propia y marcadamente distinta de los relieves que las rodean, consecuencia de su particular litología y vegetación.

En definitiva, T. coloni estaría acantonada en el extremo noroeste de la cordillera rifeña y $T$. armifrons en el Medio Atlas central y, al menos puntualmente, en el Alto Atlas, existiendo, en base a los exiguos registros existentes, una banda de separación de unos $240 \mathrm{~km}$ de anchura entre ambas. Evidentemente, se imponen nuevas prospecciones que determinen sus respectivas áreas de distribución y requerimientos ecológicos, paso previo para establecer una hipótesis sobre la biogeografía, tanto del grupo como del subgénero.

\section{AGRADECIMIENTOS}

Queremos expresar nuestro agradecimiento a las siguientes personas: D. Juan de Ferrer y D. Pablo Bercedo, por su magnífico apoyo bibliográfico; D. Francisco J. Martínez, que tanto nos ha ayudado en los muestreos faunísticos efectuados en diferentes localidades de Marruecos; Dr. José M. Ávila, que siempre nos ha facilitado el estudio de su rica colección, depositada en el Departamento de Biología Animal y Ecología de la Universidad de Granada; y por último, a D. Francisco Delgado, por la cesión de las interesantes capturas realizadas a nuestras instancias durante el transcurso de sus periplos marroquíes, as como por sus ilustrados comentarios sobre geografía de este país.

\section{Referencias}

BÁGUENA, L., 1967. Scarabaeoidea de la fauna iberobalear y pirenaica. Instituto Español de Entomología. C.S.I.C. Madrid. 567 pp.

BARAud, J., 1965. Révision du sg. Thorectes Muls. (Col. Geotrupidae). $2^{\circ}$ note: les espèces africaines. Bull. Soc. Sci. Nat. Phys. Maroc, 45: 138-156.

BARAUD, J., 1966. Révision du sous-genre Thorectes Muls. $1^{\circ}$ note: les espèces iberiques (Col. Geotrupidae). Eos, [1965], 41: 181-198.

Baraud, J., 1971. Complement au catalogue des Scarabaeoidea du Maroc. Bull. Soc. Sci. Nat. Phys. Maroc, 51: 213-220.

BARAud, J., 1977. Coléoptères Scarabaeoidea. Faune de l'Europe occidentale. Suppl. Nouv. Rev. Entomol., 7(1): $1-352$

BARAUD, J., 1985. Coléoptères Scarabaeoidea. Faune $d u$ nord de l'Afrique, du Maroc au Sinaï. Enciclopedie Entomologique, 46. Ed. Lechevalier. Paris. $652 \mathrm{pp}$.
BARAUD, J., 1992. Coléoptères Scarabaeoidea d'Europe. Faune de France, 78. Fédération française des Sociétés de Sciences naturelles et Société linnéenne de Lyon. Lyon. 856 pp.

BENABID, A., 1982. Etude phytoécologique, biogéographique et dynamique des associations et séries sylvatiques du Rif occidental (Maroc). Thèse es-sciences. Univ. Droit, Econom., Sc., Aix-Marseille III. 199 pp.

BENABID, A., 1984. Etude phytoécologique des peuplements forestiers et preforestiers du Rif centro-occidental (Maroc). Trav. Inst. Sci. Rabat, Sér. Bot., 34: $1-64$.

Benabid, A. y Fennane, M., 1994. Connaissances sur la végétation du Maroc: Phytogéographie, phytosociologie et séries de végétation. Lazaroa, 14: 21-97.

BRANCO, T., 1984. Sur le dimorphisme sexuel secondaire chez Thorectes laevigatus cobosi Baraud (Coleoptera: Geotrupidae). Entomol. Ber., Amsterdam, 44: 44-47.

Chamorro, S., 1995. El medio natural de Ceuta y su entorno: concreción y potencialidades para el desarrollo. Monografía de los Cursos de verano de la Universidad de Granada en Ceuta, IV Edición, 1994, I.E.C.-Univ. de Granada, Granada: 139-199.

Chamorro, S. y Nieto, M., 1989. Síntesis geológica de Ceuta. Ayuntamiento de Ceuta, Concejalía de Cultura, Servicio de publicaciones. Ceuta. 223 pp.

Dewhurst, C.F., 1980. Notes on some dung beetles collected in Morocco (Coleoptera, Scarabaeidae). Bull. Inst. Sci. Rabat, 4, 1979-1980: 53-68.

El Hatimi, N., Duee, G. y Hervouet, Y., 1991. La Dorsale calcaire du Hauz: ancienne marge continentale passive téthysienne (Rif, Maroc). Bull. Soc. Géol. France, 162(1): 90-97.

EscalerA, M.M. DE LA, 1914. Los Coleópteros de Marruecos. Trab. Mus. Cienc. Nat. Madrid, Ser. Zool., 11: 1-553.

GHARBAOUI, A. EL, 1981. La terre el l'homme dans la Peninsule Tingitane. Etude sur l'homme et le milieu naturel dans le Rif occidental. Trav. Inst. Sci. Rabat, Sér. Géol. Geogr. Phys., 15: 1-439.

Kocher, L., 1956. Catalogue commenté des Coléoptères du Maroc. Préface, Introduction, Carte. Trav. Inst. Sci. Chérifien, sér. zool., 7: 1-26.

Kocher, L., 1958. Catalogue commenté des Coléoptères du Maroc. VII: Lamellicornes. Trav. Inst. Sci. Chérifien, sér. zool., 16: 1-83.

Kocher, L., 1969. Catalogue commenté des Coléoptères du Maroc. Feuillets Rectificatifs. Trav. Inst. Sci. Chérifien, sér. zool., feuillet rectificatif $n^{\circ}$ 17: 1-3.

Krikken, J., 1981. Geotrupidae from the Nepal Himalayas. New flightless species of Geotrupes Latreille, 1796, with a biogeographical discussion (Insecta: Coleoptera). Senckenberg. Biol., [1980], 61(5-6): 369-381.

LÓPEZ-CoLÓN, J.I., 1981. Contribución al conocimiento del género Thorectes Mulsant, 1842 (Coleoptera Scarabaeoidea). Thorectes baraudi, nueva especie española. Nouv. Rev. Entomol., 11(3): 277-286. 
LóPEZ-CoLÓN, J.I., 1983. Contribución al conocimiento del género Thorectes Mulsant, 1842 (Coleoptera Scarabaeoidea). II. Thorectes ferreri, nueva especie española. Nouv. Rev. Entomol., 13(3): 299-303.

LÓPEZ-COLÓN, J.I., 1989. Algunas consideraciones sobre la morfología de la armadura genital masculina en el género Thorectes Mulsant, 1842 y sus implicaciones filogenéticas (Col. Scarabaeoidea, Geotrupidae). Bol. Gr. Entomol. Madrid, 4: 69-82.

LÓPEZ-Colón, J.I., 1990. Contribución al conocimiento del género Thorectes Mulsant, 1842. IX. Comentarios y nuevas notas sobre algunos Thorectes ibéricos (Coleoptera, Scarabaeoidea). Nouv. Rev. Entomol. (N.S.), 7(2): 205-207.

LÓPEZ-COLÓN, J.I., 1995. Estudio corológico de algunos Thorectes Muls. de la fauna ibero-balear y pirenaica (Coleoptera, Scarabaeoidea, Geotrupidae) (II Nota, parte I). Lambillionea, 95(2): 211-222.

LÓPEZ-COLÓN, J.I., 1996. El "Género" Thorectes Mulsant, 1842 (Coleoptera, Scarabaeoidea, Geotrupidae) en la Fauna Europea. G. It. Entomol., [1995], 7: 355-388.

LóPez-Colón, J.I., PÉrez-LóPEZ, F.J. y HeRnÁNDEZ RuIZ, 1997. Nuevos registros ibéricos de geotrúpidos de los géneros Thorectes Mulsant, 1842 y Jekelius López-Colón, 1989 (Col. Geotrupidae). Bol. Soc. Entomol. Arag., 17: 3-6.

López-COLÓn, J.I. y RoMero SAMPer, J., 1996. Estudio corológico de algunos Thorectes Muls. de la fauna ibero-balear y pirenaica (Coleoptera, Scarabaeoidea, Geotrupidae) (II Nota, parte II). Lambillionea, 96(2): 242-260.
Michard, A., 1976. Eléments de Géologie marocaine. Not. Mém. Serv. Geol. Maroc, 252: 1-408.

Quézel, R., Barbéro, M., Benabid, A., Loisel, A. y Rivas-MartínEZ, S., 1988. Contribution à l'étude des groupements préforestiers et des matorrals rifains. Ecol. Medit., 14(1-2): 77-122.

REITTER, E., 1893. Bestimmungs-Tabelle der Lucaniden und Coprophagen Lamellicornen des palaearctischen Faunengebietes. Verh. Nat. Ver. Brünn, [1892], 31: 337.

RuIZ, J.L., 1995. Los Scarabaeoidea (Coleoptera) coprófagos de la región de Ceuta (Norte de África). Aproximación faunística. Transfretana, monografía $n^{\circ}$ 2. Estudios sobre el medio natural de Ceuta y su entorno, Ceuta: 11-114.

Ruiz, J.L., Ávila, J.M. y LóPEz-Colón, J.I., 1995. Descripción de la hembra de Thorectes baraudi López-Colón, 1981 y comentarios sobre las especies del subgénero Thorectes s. str. Mulsant, 1842 (Coleoptera, Scarabaeoidea: Geotrupidae). Nouv. Rev. Entomol. (N.S.), [1994], 11(4): 365-373.

Zunino, M., 1984. Sistematica generica dei Geotrupinae (Coleoptera, Scarabaeoidea: Geotrupidae), filogenesi della sottofamiglia e considerazioni biogeografiche. Boll. Mus. Reg. Sci. Nat. Torino, 2(1): 9-162. 